\title{
Facteurs de risque pour les maladies diarrhéiques chez les enfants à Dakar : une analyse multi-niveaux avec variables latentes
}

\author{
Iulia Rautua,b ${ }^{a}$ Stéphanie Dos Santos ${ }^{b}$, Bruno Schoumaker ${ }^{c}$ \\ a Aspirant FNRS, Centre de recherche en démographie - Centre for Demographic Research, \\ Université catholique de Louvain, Belgique, iulia.rautu@uclouvain.be, +3210472957 \\ ${ }^{b}$ Laboratoire Population Environnement Développement (LPED), Institut de recherche pour le \\ développement (IRD) / Aix-Marseille université \\ ${ }^{c}$ Centre de recherche en démographie - Centre for Demographic Research, Université catholique \\ de Louvain, Belgique
}

\begin{abstract}
In Senegal, diarrheal diseases are a major burden to child health. Their network of determinants includes a wide range of factors, from different spheres and levels of analysis. The study analyzes these risk factors and their relative role in diarrheal diseases on children from Dakar. It also illustrates a new approach for synthesizing the network of these determinants. A latent class analysis (LCA) is conducted, and the resulting latent variables are subsequently used as explanatory part in a three-level logistic regression. This study confirms that the determinants of diarrhea in children belong to all three levels of analysis and that behavioral factors and neighborhood sanitation play an important role. Results also illustrate the utility of LCA in synthesizing several indicators, so as to create an integrated overall causal image, using parsimonious statistical models.
\end{abstract}

Keywords: diarrheal diseases, child health, sub-Saharan Africa, latent class analysis, multilevel models.

\section{Résumé}

Au Sénégal, les maladies diarrhéiques constituent un fardeau important, qui pèse encore lourdement sur la santé des enfants. Ces maladies sont influencées par un large éventail de facteurs, appartenant à différents niveaux et sphères d'analyse. Cet article analyse ces facteurs de risque et leur rôle relatif dans les maladies diarrhéiques de l'enfant à Dakar. Ce faisant, elle illustre une nouvelle approche pour synthétiser le réseau de ces déterminants. Une analyse en classes latentes (LCA) est d'abord menée, puis les variables latentes ainsi construites sont utilisées comme variables explicatives dans une régression logistique sur trois niveaux. Les résultats confirment que les déterminants des diarrhées chez l'enfant appartiennent aux trois niveaux d'analyse et que les facteurs comportementaux et l'assainissement du quartier jouent un rôle prépondérant. Les résultats illustrent aussi l'utilité des LCA pour synthétiser plusieurs indicateurs, afin de créer une image causale intégrée, tout en utilisant des modèles statistiques parcimonieux.

Mots-clés: maladies diarrhéiques, santé des enfants, Afrique subsaharienne, modèles à classes latentes, analyses multiniveaux.

\section{Introduction}

Au Sénégal, les maladies diarrhéiques constituent l'un des problèmes de santé publique les plus importants. En 2010, elles étaient estimées comme étant la première cause de mortalité juvénile $(18 \%$ des décès chez les enfants de moins de 5 ans) et la deuxième cause de décès chez les chez les enfants de 5 à 14 ans, avec $7,5 \%$ des décès (Institute for Health Metrics and Evaluation, 20/3). Dans la capitale, Dakar, l'urbanisation rapide des trois dernières décennies a conduit à une forte densité de population, dans un contexte de comportements d'hygiène souvent inadéquats, d'un manque d'assainissement et de politiques de santé parfois peu efficaces (Sambe-Ba et al., 2013). La croissance urbaine parfois non planifiée a créé un espace qui se caractérise par une importante hétérogénéité en termes de conditions de vie, où $36 \%$ des ménages ne disposent pas d'installations sanitaires améliorés et trois quarts ne sont pas connectés au réseau d'égouts (Scott et al., 20I3). Cette population, et notamment les enfants qui sont particulièrement vulnérables, se 
retrouve davantage exposée à des risques de contracter des maladies diarrhéiques, en raison d'une transmission qui se fait le plus souvent via de l'eau contaminée. Une transmission via l'eau signifie aussi que l'éventail des facteurs de risque est très varié et susceptible d'appartenir à différentes sphères et différents niveaux, étant donné l'ubiquité de l'eau dans la vie quotidienne (Fewtrell and Colford, 2004). La plupart des études menées en Afrique subsaharienne sur les maladies diarrhéiques chez les enfants se sont concentrées sur les enfants de moins de 5 ans résidant dans des zones rurales (Sambe-Ba et al., 20l3). Ainsi, il existe un certain décalage entre l'abondance de littérature existante sur les diarrhées infantiles et juvéniles en milieu rural et une certaine méconnaissance concernant les facteurs qui favorisent ces infections chez les enfants plus âgés, ou ceux résidant en ville. Pourtant, la prévalence des diarrhées dans les villes d'Afrique subsaharienne est similaire à celle du milieu rural (Boschi-Pinto et al., 2006) et aujourd'hui, l'urbanisation rapide fait qu'une proportion importante de la population réside en ville : au Sénégal, près de la moitié (plus de $48 \%$ ) de la population est considérée comme urbaine (United Nations Human Settlements Programme, 2007). Cela signifie que les diarrhées en milieu urbain sont très nombreuses et elles constituent un fardeau de santé publique important, au moins en termes d'effectifs absolus. Par ailleurs, le réseau de facteurs de risque est susceptible d'être plus varié en ville en raison d'une plus grande diversité des styles de vie et de l'hétérogénéité de l'environnement urbain. En particulier, dû à l'importante croissance urbaine , une proportion grandissante de la population urbaine vit dans des slums (habitats informels). Au Sénégal, cette population a presque doublé entre 1990 et 2005 ; elle est passée de 2.276 .000 à 4.181.000 habitants (United Nations Human Settlements Programme, 2007). Les risques particuliers liés aux infections diarrhéiques encourus par cette population sont susceptibles d'être différents de ceux qui caractérisent le milieu rural. Chez les enfants, une fois le rôle protecteur de l'allaitement disparu, ils deviennent plus vulnérables à l'infection aux pathogènes concernés (Khatab and Kandala, 20II; Woldemicael, 20I I).

L'objectif principal de cet article est d'analyser le rôle relatif des déterminants de la diarrhée chez l'enfant dans une grande agglomération urbaine, la capitale du Sénégal, en distinguant la part des facteurs relatifs au niveau de l'individu, de sa famille ainsi qu'au niveau de son environnement proche. Ce faisant, nous illustrerons une approche statistique relativement nouvelle en démographie, qui permet de synthétiser le réseau complexe de déterminants dans un contexte d'hétérogénéité sociale et spatiale.
L'analyse en classes latentes (LCA) permet de regrouper un large éventail de déterminants, de les synthétiser dans des dimensions plus larges, afin de créer une image causale complète, sans alourdir l'analyse statistique.

\section{Revue de la littérature}

De nombreux facteurs ont été associés à la transmission des maladies diarrhéiques, y compris certains comportements, conditions de vie et caractéristiques de l'environnement proche (Woldemicael, 20l I). L'hygiène personnelle, de I'habitation et des aliments est souvent considérée comme un des principaux déterminants de la transmission des pathogènes (Alirol et al., 20ll). L'adoption de certains comportements en la matière dépend des caractéristiques individuelles telles que le niveau de vie et l'éducation.

Par ailleurs, certaines caractéristiques de l'environnement domestique peuvent faciliter ou empêcher la propagation des pathogènes: dans les ménages sans eau courante, l'eau stockée durant de longues périodes présente plus de risque de contamination (Fewtrell and Colford, 2004). Les installations sanitaires inadéquates représentent également des voies de contamination avec des matières fécales (Barreto et al., 2007). De même, le stockage des ordures ménagères dans l'habitation peut aussi constituer un facteur de risque, tant en termes de durée, qu'en termes de récipient utilisé.

En outre, le risque encouru par un ménage qui ne dispose pas d'équipements et d'installations appropriés s'étend souvent aussi aux ménages voisins, qui se retrouvent plus exposés à des contacts avec des eaux sales ou des ordures ménagères (Heller, 1999). Ainsi, l'environnement du quartier - bien qu'ayant un impact moins explicite que celui offert par le ménage - constitue un niveau où peuvent se manifester des facteurs de risque, à travers le manque de salubrité. La présence d'eaux usagées et de déchets ou d'excréments augmente le risque de prolifération des mouches. Cela, à son tour, facilite la transmission des pathogènes responsables de diarrhées, dans les populations vivant à proximité (Rego et al., 2005).

Les facteurs qui peuvent influencer les maladies diarrhéiques sont donc nombreux, et ils appartiennent à plusieurs sphères et sont interconnectés. Ceci complique l'analyse car les régressions classiques ne permettent pas de prendre en compte de nombreuses variables explicatives. Conséquemment, la majorité des analyses se concentrent sur une seule sphère (le rôle des comportements ou de l'environnement proche) ou un seul niveau d'analyse (celui du ménage ou celui de 
l'aire géographique), ce qui accroît le risque de facteurs confondants. Aussi, les approches classiques reposent sur une hypothèse d'indépendance (entre individus du même ménage, ou les ménages du même quartier), ce qui est souvent contredit par les données et sous-estime les erreurs standard des coefficients.

Le cadre de cette analyse définit donc trois niveaux (Figure I): (I) celui de l'individu, qui prend en compte les caractéristiques bio-démographiques de l'enfant; (2) celui des ménages, qui regroupe à la fois des caractéristiques sociales, comportementales et de conditions de vie; et (3) celui du quartier, qui caractérise son assainissement. Il considère en outre deux mécanismes de transmission des maladies diarrhéiques: d'un côté, la vulnérabilité liée aux facteurs individuels et de l'autre, l'exposition aux pathogènes.

Figure I: Cadre d'analyse

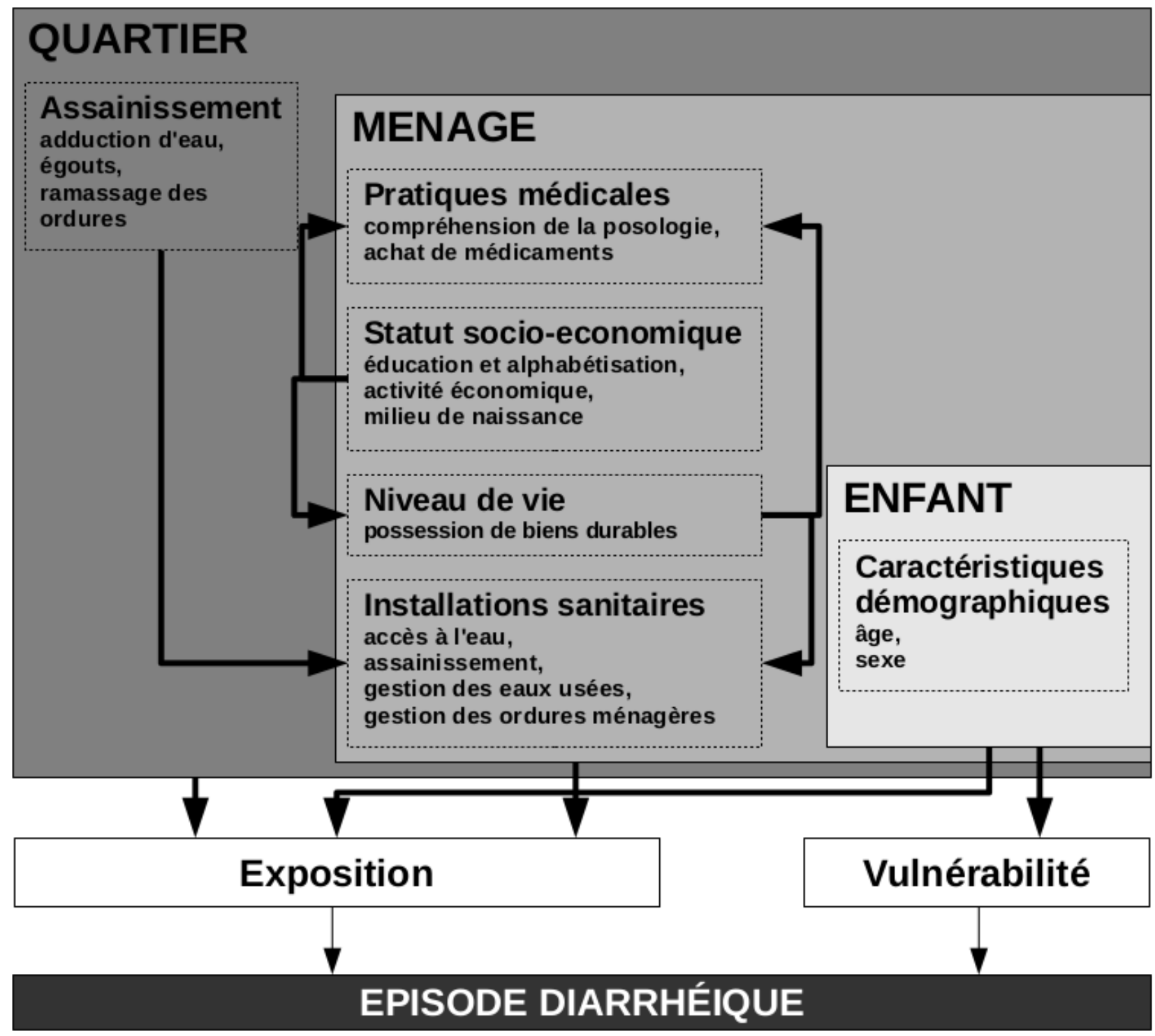




\section{Données et Méthodes \\ Données}

Cet article se base sur les données quantitatives issues du projet "Paludisme et diversité de l'environnement urbain africain : un enjeu majeur pour la mise en place des thérapies à base d'artémisinine (ACT)" (ACTU-PALU). Ce projet, ciblé sur Dakar et sa banlieue, a été coordonné par I'Institut de Recherche pour le Développement (IRD), en collaboration avec l'Université Cheikh Anta Diop de Dakar. L'enquête par questionnaire a été menée entre le 15 septembre et le 22 décembre 2008, dans 50 quartiers' et sur un échantillon représentatif de 2952 ménages, avec 7416 enfants de 2 à 10 ans.

L'enquête a utilisé trois volets. Le premier a recueilli des informations liées au quartier, auprès d'informateurs (entre 4 et II selon le quartier), dont le chef de quartier ou son délégué. Ce volet collecte des caractéristiques de l'environnement, des infrastructures et des réseaux sociaux dans le quartier. Un deuxième volet se réfère aux ménages et a été administré à un membre permanent du ménage (souvent le chef du ménage ou sa conjointe). $\mathrm{Ce}$ volet contient des informations sur chaque membre du ménage, ainsi que sur les caractéristiques et niveau de vie du ménage. Le troisième volet, administré à une mère ou tutrice d'un enfant de 2 à 10 ans, regroupe des caractéristiques sociodémographiques et culturelles, ainsi que des informations sur les comportements en matière d'hygiène.

L'enquête renseigne donc à la fois des caractéristiques socio-démographiques, comportementales, sur le logement et sur la zone de résidence. Elle renseigne aussi l'occurrence d'un épisode diarrhéique chez les enfants du ménage (Lalou, 2008). Cela constitue la variable d'intérêt de cette recherche. Elle a été définie selon le modèle de I'OMS, c'est-à-dire comme étant l'émission d'au moins trois selles molles ou liquides par jour, avec une période de référence de 15 jours.

\section{Méthodes}

La structure hiérarchique des données (individu, ménage, quartier) permet une approche statistique multi-niveaux, qui prend en compte la nonindépendance des individus au sein d'un même groupe et permet de distinguer l'effet des facteurs intervenant sur ces trois niveaux d'observation. Certains de ces facteurs renvoient à des concepts multidimensionnels, comme le statut socioéconomique, le niveau de vie du ménage ou

1 Dans l'enquête, les quartiers regroupent, en moyenne, 170 concessions et 267 ménages; le taux de réponse a été de $96 \%$ au niveau des concessions et $98 \%$ au niveau des ménages l'environnement domestique, qui influent sur la capacité à mettre en œuvre des mesures préventives de la maladie (Mosley and Chen, 1984). Ils ne peuvent pas être mesurés directement ou correctement approximés par aucun indicateur isolément. Cependant, ils peuvent être estimés, en regroupant plusieurs variables manifestes pertinentes dans des indicateurs.

A cet effet, l'étude emploie des variables latentes sous la forme d'analyse en classes latentes (LCA). L'utilisation de la LCA produit des modèles parcimonieux, en synthétisant une grande quantité de données (appelées "variables manifestes") dans un nombre prédéfini de catégories (appelées " classes"). Comparé à d'autres méthodes de réduction de dimensions - comme l'analyse en composantes principales (ACP) - un avantage de la LCA est qu'elle ne requiert pas des données continues et peut donc être utilisée avec des données catégorielles (McCutcheon, 1987).

Un autre avantage est que LCA utilise des probabilités conditionnelles, tandis que I'ACP utilise une transformation orthogonale. Cet aspect - ainsi que le fait que le nombre de classes est déterminé par le chercheur - signifie qu'elle peut être entièrement harmonisée au cadre théorique de la recherche et que les résultats sont facilement interprétables.

Les variables latentes estimées portent sur le statut socio-économique de la mère/tutrice de l'enfant, les comportements en matière de santé adoptées dans le ménage, le niveau de vie et l'appareil sanitaire du ménage, ainsi que le système sanitaire et de salubrité du quartier. Comme la LCA permet au chercheur d'établir le nombre de classes estimées, nous avons appliqué successivement trois critères, afin de choisir le nombre de classes. Le premier a été celui d'adaptation au cadre théorique et à la revue de littérature. Ensuite, entre plusieurs modèles similaires en termes d'intérêt, nous avons comparé les valeurs du critère bayesien $\left(\mathrm{BIC}^{2}\right)$ et, finalement, nous avons appliqué un critère de parcimonie (le plus réduit nombre de classes, qui permet un modèle bien identifié). Les variables latentes ainsi estimées contiennent deux classes chacune et elles ont été estimés à l'aide du package poLCA du logiciel $\mathrm{R}$ (Linzer and Lewis, 20I I).

Afin d'estimer le "statut socio-économique de la mère/tutrice ", quatre variables catégorielles liées à l'alphabétisation, au niveau d'éducation, à l'activité professionnelle et au milieu de naissance ont été utilisées.

2 Le critère le plus souvent utilisé dans l'évaluation des modèles à classes latentes 
La variable latente comportementale estimée en tant que proxy des mesures de santé et d'hygiène dans le ménage se réfère aux "pratiques médicales". Pour la construire, nous avons utilisé cinq items dichotomiques, liés aux connaissances et comportements médicaux des mères/tutrices enquêtées tels l'automédication, la bonne compréhension et l'observance des prescriptions médicales.

La variable latente "niveau de vie du ménage » a été estimée en utilisant des indicateurs de possession de biens durables (Filmer and Pritchett, 200I), avec onze variables dichotomiques liées à la possession de différents équipements et biens durables dans le ménage.

Quant à la variable latente «installations sanitares " du ménage, elle a été construite sur base de six variables catégorielles mesurant l'accès à l'eau, le type de toilettes et l'évacuation des eaux usées et des déchets ménagers.

Au niveau du quartier, nous avons inclus à la fois des indicateurs objectifs et subjectifs pour construire la variable "assainissement du quartier». Les données de nature objective dans l'enquête se limitent à la couverture des infrastructures sanitaires et de salubrité. Pourtant, ce type d'indicateurs souvent collecté, publié et cité - ne prend pas en compte beaucoup d'autres facteurs, tels la quantité, la qualité et le prix d'utilisation de ces infrastructures (United Nations Human Settlements Programme, 2007). Ainsi, ils offrent une image biaisée et souvent surestiment la qualité de ces infrastructures dans les villes des pays en développement (Heller, 1999). Afin de tenir compte de ces aspects, nous avons inclus trois indicateurs objectifs dichotomiques mesurant la couverture des réseaux d'adduction d'eau, d'égouts et du service de ramassage des ordures, ainsi que quatre indicateurs subjectifs dichotomiques concernant la qualité de ces réseaux perçue par les résidents.

Les indicateurs utilisés pour l'estimation des variables latentes (ainsi que la variable dépendante de l'analyse), se caractérisent par une faible proportion de valeurs manquantes, de moins de I,5\%. La LCA permet de prendre en compte ces valeurs manquantes, et l'information utilisée reprend donc l'ensemble des cas.

Etant donné que les indicateurs manifestes utilisés pour créer ces variables latentes constituent des déterminants pertinents pour les maladies diarrhéiques, les variables latentes peuvent ellesmêmes être considérées comme des déterminants potentiels pour ces maladies diarrhéiques. Ainsi, certaines des classes issues de la LCA peuvent être traitées comme étant plus à risque que d'autres, en fonction de leurs probabilités conditionnelles, qui relient chaque classe estimée aux indicateurs manifestes (Tableau I).

Dans chacun des cinq modèles élaborés, les unités d'analyse (individu, ménage ou quartier, selon la variable latente estimée) sont affectées à leurs classes latentes respectives, en fonction de ces probabilités conditionnelles. La proportion des unités assignées à chaque classe correspond à la distribution des classes dans la population. Celle-ci est déterminée par le modèle et permet de dresser un portrait de l'échantillon, selon les variables latentes estimées. Ainsi, les classes latentes estimées peuvent être utilisées dans d'autres analyses statistiques. Dans cette recherche, ces variables latentes ont été utilisées en tant que partie explicative dans une régression logistique multi-niveaux, ayant comme variable dépendante l'épisode diarrhéique renseigné.

Une approche multi-niveaux permet de comprendre où et comment les effets surviennent et donc de distinguer le rôle de chaque niveau d'analyse. L'ordonnée à l'origine et/ou la pente d'une régression multi-niveaux peuvent varier à travers les groupes et la variance est calculée à tous les niveaux d'analyse. Ainsi, dans un modèle vide (sans variable explicative), le coefficient de corrélation intraclasse (ICC) indique la proportion du total de la variance dans la variable dépendante qui est expliquée par chaque niveau.

Cette étude emploie une régression sur trois niveaux: individuel (l'enfant), le ménage et le quartier. La régression utilise une fonction logit et a été exécutée sous MlwiN (Rasbash et al., 2009), sans pondérations ${ }^{3}$, sur les 7416 enfants, vivant dans les 2952 ménages des 50 quartiers.

\section{Résultats}

Parmi les 74I6 enfants de l'échantillon, 3772 avaient cinq ans ou moins au moment de l'enquête et 3644 avaient entre 6 et 10 ans révolus. Du total des enfants, II5I (I5,52 \%) ont eu une diarrhée dans les 15 jours précédant l'enquête, $6160(83,06 \%)$ n'ont pas eu la diarrhée et pour 105 enfants $(I, 42 \%)$ cette information est manquante. Parmi les $\mid 151$ enfants qui ont subi un épisode diarrhéique renseigné, 588 (5I\%) sont des garçons et 563 (49\%) des filles, 764 (deux tiers) avaient cinq ans ou moins et 387 (un tiers) entre six et dix ans.

\section{Caractéristiques de la population: analyse des classes latentes}

L'analyse des probabilités conditionnelles des cinq variables latentes estimées (auxquelles correspondent dix classes) révèle que la Classe $A$ regroupe des

$3 \quad$ Une analyse avec pondérations a été également testée mais elle ne modifie pas substantiellement pas les résultats. 
mères/tutrices avec un niveau socio-économique haut, tandis que la Classe $B$ exprime un niveau socioéconomique plus bas (Tableau I). Au niveau du ménage, les mères/tutrices de la Classe $C$ sont plus susceptibles d'adopter des pratiques médicales adéquates (par exemple, l'achat de médicaments dans des pharmacies agréées), tandis que la Classe $D$ exprime des pratiques médicales inadéquates. Les ménages assignés à la Classe $E$ sont plus aisés que ceux assignés à la Classe F. L'appartenance à la Classe $\mathrm{H}$ est associée à de meilleurs équipements sanitaires dans le ménage, comparé à la Classe G. Au niveau du quartier, ceux assignés à la Classe I sont caractérises par de meilleures infrastructures d'assainissement, comparés à ceux assignés à la Classe J.

La répartition des ménages et quartiers au sein des différentes classes ainsi créées permet de créer un " portrait » de la population de l'étude. Ainsi, $47 \%$ des mères/tutrices se caractérisent par un statut socio-économique plus haut, contre $53 \%$ avec un statut plus bas (classe B). Trois quarts $(75 \%)$ des ménages adoptent des comportements de santé adéquats. En termes de niveau de vie, la répartition des ménages entre les deux classes $E$ et $F$ est quasiéquivalente, avec $49 \%$ des ménages considérés comme plus aisés. $57 \%$ des ménages ont des installations sanitaires déficientes. Finalement, un tiers des quartiers (34\%) se distinguent par une meilleure qualité des infrastructures d'assainissement. Ceci dit, vu que ces variables latentes ne sont pas associées les unes aux autres, le fait d'appartenir à une classe d'une variable latente n'indique pas l'appartenance à une certaine classe dans une autre variable latente. Donc une observation peut se trouver dans une classe favorable en ce qui concerne, par exemple, les " installations sanitaires", mais défavorable en ce qui concerne les «pratiques médicales ».

Tableau I : Indicateurs, probabilités conditionnelles et distribution des classes dans les variables latentes

\begin{tabular}{|c|c|c|c|}
\hline \multicolumn{2}{|l|}{ Statut socio-économique de la mère $(\mathrm{N}=4024$; $\mathrm{BIC}$ : 22796) } & \multirow{2}{*}{$\begin{array}{l}\text { Classe A } \\
(47 \%) \\
0,00\end{array}$} & \multirow{2}{*}{$\begin{array}{l}\text { Classe B } \\
(53 \%) \\
0,78\end{array}$} \\
\hline \multirow{3}{*}{ Niveau d'éducation } & Sans éducation formelle & & \\
\hline & Primaire & 0,55 & 0,22 \\
\hline & Secondaire ou plus & 0,45 & 0,00 \\
\hline \multirow{3}{*}{ Statut d'activité } & Travaille & 0,45 & 0,48 \\
\hline & Femme au foyer & 0,45 & 0,49 \\
\hline & Autre & 0,09 & 0,03 \\
\hline \multirow{3}{*}{ Milieu de naissance } & Urbain & 0,89 & 0,68 \\
\hline & Rural & 0,08 & 0,28 \\
\hline & A l'étranger & 0,03 & 0,04 \\
\hline \multirow{2}{*}{ Alphabétisation } & Non & 0,01 & 0,97 \\
\hline & Oui & 0,99 & 0,03 \\
\hline \multicolumn{2}{|l|}{ Pratiques médicales (N = 2952 ; BIC : I I758) } & $\begin{array}{l}\text { Classe C } \\
(75 \%)\end{array}$ & $\begin{array}{l}\text { Classe D } \\
(25 \%)\end{array}$ \\
\hline \multirow{2}{*}{$\begin{array}{l}\text { A donné à l'enfant un médicament inconnu et non } \\
\text { recommandé par un professionnel de la santé }\end{array}$} & Non & 0,77 & 0,49 \\
\hline & Oui & 0,23 & 0,51 \\
\hline \multirow{2}{*}{$\begin{array}{l}\text { Comprend les instructions pour prendre les } \\
\text { médicaments }\end{array}$} & Avec difficulté & 0,20 & 0,25 \\
\hline & Facilement & 0,80 & 0,75 \\
\hline \multirow{2}{*}{$\begin{array}{l}\text { Achète des médicaments dans les pharmacies par } \\
\text { terre/vendeurs ambulants }\end{array}$} & Non & 0,97 & 0,16 \\
\hline & Oui & 0,03 & 0,84 \\
\hline \multirow{2}{*}{ Achète des médicaments au marché } & Non & 0,96 & 0,32 \\
\hline & Oui & 0,04 & 0,68 \\
\hline \multicolumn{2}{|l|}{ Niveau de vie du ménage ( $N=2952$; BIC : 27478) } & $\begin{array}{l}\text { Classe E } \\
(49 \%)\end{array}$ & $\begin{array}{l}\text { Classe F } \\
(51 \%)\end{array}$ \\
\hline \multirow{2}{*}{ Radio } & Non & 0,08 & 0,30 \\
\hline & Oui & 0,92 & 0,70 \\
\hline \multirow{2}{*}{ Téléviseur } & Non & 0,00 & 0,28 \\
\hline & Oui & $\mathrm{I}, 00$ & 0,72 \\
\hline \multirow{2}{*}{ Vidéo (DVD ou VCD) } & Non & 0,23 & 0,82 \\
\hline & Oui & 0,77 & 0,18 \\
\hline \multirow{2}{*}{ Téléphone fixe } & Non & 0,58 & 0,98 \\
\hline & Oui & 0,42 & 0,02 \\
\hline
\end{tabular}




\begin{tabular}{|c|c|c|c|}
\hline \multicolumn{2}{|c|}{ Statut socio-économique de la mère ( $N=4024$; BIC : 22796) } & \multirow{2}{*}{$\begin{array}{l}\text { Classe A } \\
(47 \%) \\
0,88\end{array}$} & \multirow{2}{*}{$\begin{array}{l}\begin{array}{l}\text { Classe B } \\
(53 \%)\end{array} \\
0,99\end{array}$} \\
\hline Cuisiniòro & Non & & \\
\hline Cuisiniere au gaz ou electrique & Oui & 0,12 & 0,01 \\
\hline \multirow{2}{*}{ Salon } & Non & 0,27 & 0,88 \\
\hline & Oui & 0,73 & 0,12 \\
\hline \multirow{2}{*}{ Ordinateur } & Non & 0,81 & 1,00 \\
\hline & Oui & 0,19 & 0,00 \\
\hline \multirow{2}{*}{ Refrigérateur } & Non & 0,32 & 0,88 \\
\hline & Oui & 0,68 & 0,12 \\
\hline \multirow{2}{*}{ Congélateur } & Non & 0,86 & 0,99 \\
\hline & Oui & 0,14 & 0,01 \\
\hline \multirow{2}{*}{ Ventilateur } & Non & 0,09 & 0,50 \\
\hline & Oui & 0,91 & 0,50 \\
\hline \multirow{2}{*}{ Voiture } & Non & 0,80 & 0,98 \\
\hline & Oui & 0,20 & 0,02 \\
\hline \multicolumn{2}{|l|}{ Installations sanitaires (N = 2952; BIC : 23036) } & $\begin{array}{l}\text { Classe G } \\
(57 \%)\end{array}$ & $\begin{array}{l}\text { Classe H } \\
(43 \%)\end{array}$ \\
\hline \multirow{3}{*}{ Mode d'approvisionnement en eau du ménage } & Robinet à l'extérieur & 0,15 & 0,14 \\
\hline & Robinet à l'intérieur & 0,67 & 0,83 \\
\hline & Autre & 0,18 & 0,04 \\
\hline \multirow{3}{*}{ Recours à un autre mode d'approvisionnement en eau } & Non & 0,64 & 0,76 \\
\hline & Oui & 0,36 & 0,24 \\
\hline & WC relié à l'égout & 0,05 & 0,69 \\
\hline \multirow[t]{2}{*}{ Type de toilettes } & WC non relié à l'égout & 0,55 & 0,12 \\
\hline & Autre & 0,40 & 0,19 \\
\hline \multirow{2}{*}{ Toilettes réservées au ménage } & Oui & 0,59 & 0,68 \\
\hline & Non & $0,4 \mathrm{I}$ & 0,32 \\
\hline \multirow{2}{*}{ Gestion des eaux ménagères } & Egout / fosse & 0,06 & 1,00 \\
\hline & Autre & 0,94 & 0,00 \\
\hline \multirow{2}{*}{ Gestion des ordures ménagères } & Poubelle & 0,84 & 0,97 \\
\hline & Décharge ailleurs & 0,16 & 0,03 \\
\hline \multicolumn{2}{|l|}{ Assainissement du quartier $(\mathrm{N}=50 ; \mathrm{BIC}: 407)$} & $\begin{array}{l}\text { Classe I } \\
(34 \%)\end{array}$ & $\begin{array}{l}\text { Classe J } \\
(66 \%)\end{array}$ \\
\hline \multirow{2}{*}{ Couverture du réseau d'adduction d'eau fonctionnel } & Complète & 0,94 & 0,91 \\
\hline & Incomplète & 0,06 & 0,09 \\
\hline \multirow{2}{*}{ Couverture du réseau d'égouts } & Complète & 0,85 & 0,00 \\
\hline & Incomplète & 0,15 & 1,00 \\
\hline \multirow{2}{*}{ Couverture du service public de ramassage d'ordures } & Complète & 0,88 & 0,42 \\
\hline & Incomplète & 0,12 & 0,58 \\
\hline \multirow{2}{*}{ Coupures d'eau } & Souvent & 0,35 & 0,52 \\
\hline & Rarement & 0,65 & 0,48 \\
\hline \multirow{2}{*}{ Qualité perçue de l'accès à l'eau } & Bonne & 1,00 & 0,75 \\
\hline & Mauvaise & 0,00 & 0,25 \\
\hline \multirow{2}{*}{ Qualité perçue de l'assainissement } & Bonne & 0,79 & 0,00 \\
\hline & Mauvaise & 0,21 & 1,00 \\
\hline \multirow{2}{*}{ Qualité perçue de la gestion des déchets } & Bonne & 0,66 & 0,51 \\
\hline & Mauvaise & 0,34 & 0,49 \\
\hline
\end{tabular}

Facteurs de risque pour les diarrhées: analyse multi-niveaux

Un des intérêts des modèles multi-niveaux est de pouvoir identifier la part de la variance dans la variable d'intérêt qui est expliquée par chaque niveau d'observation. Dans le modèle vide, la variance au niveau ménage était de 0,966 et au niveau du quartier, de 0,033 . Ainsi, le coefficient de corrélation intraclasse (Tableau 2), montre que presqu'un quart $(23 \%)$ de la variance dans la variable expliquée se 
trouve au niveau du ménage, tandis que seulement $1 \%$ de la variance est liée au quartier. Cela n'est pas surprenant, étant donné que la plupart de la variance se trouve aux niveaux inférieurs (individu et ménage). Un autre aspect qui pourrait contribuer au faible pouvoir d'explication du niveau "quartier» est le nombre assez restreint d'observations à ce niveau, avec seulement 50 quartiers dans l'échantillon.

Concernant les variables explicatives, sur les deux variables démographiques, seul l'âge de l'enfant a un impact sur les diarrhées, agissant comme un léger facteur de protection. En ce qui concerne la mère ou tutrice de l'enfant, ni son âge, ni son statut socioéconomique ne semblent avoir un impact significatif sur l'occurrence d'un épisode diarrhéique chez l'enfant. Quant au statut socio-économique, un niveau plus haut est généralement perçu comme un facteur de protection contre les infections, tandis que dans nos résultats, il n'a pas d'impact significatif. Afin de tester une possible association, nous avons aussi vérifié l'hypothèse que la variable "pratiques médicales " puisse saisir une partie de l'effet du statut socio-économique, mais le résultat se maintient, même en enlevant la variable "pratiques médicales " du modèle (résultats non présentés).

Les facteurs de nature comportementale (la variable latente "pratiques médicales") et le niveau de vie du ménage ont un impact statistiquement significatif sur l'occurrence des épisodes de diarrhées : les odds ratios des diarrhées sont $30 \%$ à $32 \%$ plus élevés chez les enfants qui proviennent de ménages pauvres ou de ménages où les pratiques médicales sont inadéquates.

$\mathrm{Si}$, au niveau du ménage, la variable latente « installations sanitaires» ne joue pas un rôle significatif sur la diarrhée chez l'enfant, au niveau du quartier, une mauvaise qualité perçue et la couverture incomplète de l'infrastructure sanitaire et de salubrité (variable latente "assainissement du quartier ») ont un impact négatif sur la maladie.

Tableau 2 : Facteurs de risque pour les maladies diarrhéiques (modèle sur trois niveaux à variance composée)

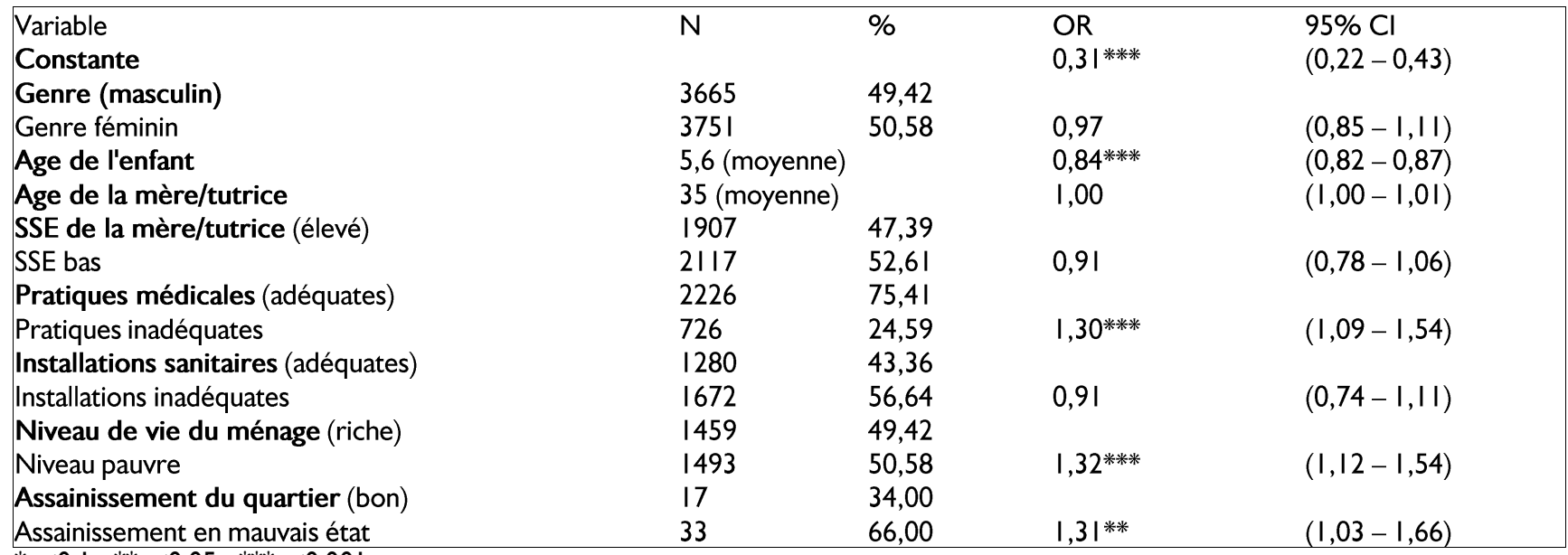

Assainissement en mauvais éta

${ }^{*} \mathrm{p}<0.1$; ** $\mathrm{p}<0.05 ;{ }^{* * *} \mathrm{p}<0.00$ I

ICC quartier : 0,0 I

ICC ménage : 0,23

\section{Discussion}

Les résultats de notre analyse indiquent que les diarrhées chez l'enfant sont influencées à la fois par des facteurs liés à l'individu, au ménage et au quartier. Cela confirme l'hypothèse d'un large éventail de facteurs, dans différents domaines, qui contribuent au risque d'infection. Au niveau de l'individu, l'âge de l'enfant influe significativement sur les maladies diarrhéiques. Cela peut être dû à deux mécanismes d'action. D'un côté, les comportements des enfants évoluent beaucoup avec l'âge, ce qui peut agir sur les niveaux d'exposition. De l'autre côté, avec l'âge, le système immunitaire de l'enfant se développe, ce qui le rend moins vulnérables (Kandala et al., 2006).
Les résultats mettent en évidence le rôle joué par les comportements préventifs dans la santé des enfants. L'impact de variable latente "pratiques médicales» est considérable et cela, indépendamment de la composante socioéconomique. La composante comportementale ressort ainsi de façon prégnante et souligne l'utilité des campagnes de sensibilisation et d'éducation de la population à risque. Selon certains auteurs, ce type d'actions, surtout si elles sont ciblées sur le lavage des mains, pourrait avoir des résultats significatifs même dans un contexte d'équipements sanitaires déficients (Fewtrell and Colford, 2004). 
Dans cette étude, le statut socio-économique de la mère n'agit pas significativement sur les probabilités d'occurrence d'épisodes diarrhéiques chez l'enfant. D'une part, des aspects liés à la garde d'enfants sont susceptibles d'interagir avec le statut socioéconomique de la mère ou tutrice et diminuer son impact positif (Hobcraft, 1993). D'autre part, ce résultat pourrait être lié au fait que les femmes mieux éduquées sont plus enclines à prêter attention à la santé de leurs enfants que les mères sans instruction. La diarrhée chez l'enfant a été enregistrée sur base de déclarations lors de l'enquête, et les données pourraient donc être biaisées par une tendance à sous-déclarer ces épisodes de façon disproportionnée dans les familles moins éduquées. II faut toutefois noter que la diarrhée est un symptôme facilement identifiable par les parents et que la période de référence ne dépasse pas celle qui est considérée comme procurant des réponses précises (Fewtrell and Colford, 2004).

Contrairement aux installations sanitaires domestiques, l'infrastructure sanitaire et de salubrité du quartier exerce un impact significatif sur les maladies diarrhéiques. Le manque d'assainissement du quartier peut donc annuler un potentiel effet bénéfique des équipements du ménage : une stratégie inadéquate pour se débarrasser des eaux usées ou des déchets ménagers, adoptée par certains ménages (décharge sauvage), augmentera le risque d'infection chez la population vivant à proximité du dépotoir, plutôt que chez la population qui l'adopte (Heller, 1999). Le manque d'investissements dans les infrastructures sanitaires et de salubrité aurait donc un impact indirect même sur la population qui n'est pas directement concernée. Afin de mieux capter l'impact de l'assainissement au niveau du quartier, en plus des indicateurs de couverture, nous avons également inclus des indicateurs relatifs à la qualité perçue de celui-ci. II faut pourtant tenir compte du fait que les informations subjectives ne sont pas nécessairement représentatives de l'opinion de la population totale du quartier. Une étude qualitative pourrait apporter plus d'informations sur ce volet de l'enquête.

Aussi, certaines variables qui pourraient influencer le risque de diarrhées n'ont pas été recueillies dans l'enquête et donc n'ont pas été inclues dans l'analyse. Une variable absente de nos analyses qui joue probablement un rôle important est le statut d'allaitement. L'enquête s'est limitée aux enfants de 2 à 10 ans, en partie pour éviter cet effet confondant, puisque, contrairement au milieu rural, l'allaitement des enfants de deux ans ou plus en milieu urbain au Sénégal est une pratique beaucoup moins courante (Gartner et al., 2007). Ainsi, le biais introduit par l'absence de prise en compte de cette variable n'est probablement pas important. Cependant, certaines informations non recueillies par l'enquête, liées à la fratrie ou au poids à la naissance, pourraient être envisagées dans le cadre de plus amples recherches.

Finalement, sur un plan méthodologique, il faut souligner l'intérêt de l'analyse en classes latentes pour réduire le nombre de variables considérées. Le modèle utilisé ici comprend huit paramètres: cinq variables latentes et trois indicateurs démographiques individuels. Une régression logistique multiniveaux classique sur ces données ${ }^{4}$ qui inclurait séparément chaque indicateur pertinent et susceptible d'influer sur la maladie, en base de la littérature scientifique existante, comprendrait 35 variables explicatives. Cela générerait des coefficients non statistiquement significatifs, en raison de la multi-colinéarité et d'un rapport trop élevé entre le nombre de modalités et le nombre de cas.

\section{Conclusion}

Les objectifs principaux de cette étude étaient d'analyser le réseau de facteurs de risque pour les maladies diarrhéiques chez les enfants à Dakar, ainsi que leur importance respective. Afin d'obtenir des résultats robustes et fiables, nous avons pris en compte plusieurs sphères et niveaux d'analyse. $\mathrm{Ce}$ faisant, notre recherche illustre l'utilité de la LCA pour les études dans le domaine de la santé publique, par l'élaboration de modèles complexes, mais facilement estimables et interprétables. Une analyse statistique en deux étapes a été effectuée ici: cinq variables latentes ont été créées, en utilisant une LCA, puis ces variables latentes ont été utilisées dans une régression logistique multi-niveaux, ayant comme variable dépendante l'occurrence d'un épisode diarrhéique chez l'enfant.

Les résultats montrent que l'utilisation de la LCA, en conjonction avec une analyse-multi-niveaux, permet d'intégrer des caractéristiques démographiques, socio-économiques, comportementales et environnementales, afin de créer une image causale complexe sur les risques de maladie, tout en utilisant des modèles statistiques parcimonieux.

Parmi les facteurs de risque identifiés, le rôle notable des pratiques de santé et de l'assainissement au niveau du quartier est particulièrement probant. Ces deux facteurs ont un effet distinct du niveau d'éducation et des ressources économiques de la famille. Ceci signifie que des progrès importants dans la lutte contre les maladies diarrhéiques peuvent être faits via des campagnes ciblées d'éducation et de Résultats non présentés 
motivation de la population, corroborées par des interventions de base en termes d'assainissement.

Ce type d'interventions, qui ne requiert pas d'actions liées au niveau général d'éducation ou à la prospérité de la population, est donc moins coûteux. Les résultats montrent que même indépendamment du statut-socio-économique, ces mesures simples peuvent avoir un impact significatif et conduire à une diminution nette des maladies diarrhéiques, qui constituent encore une des plus grandes causes de décès chez les enfants en Afrique subsaharienne.

\section{Remerciements}

Les auteurs souhaitent remercier le Prof. Dr. Allan L. McCutcheon pour son aide précieuse en méthodes statistiques les organismes qui ont financé cette recherche, le projet Horizon 2020 de l'Union Européenne, sous le grant Marie Skłodowska-Curie No 690984: DEMOSTAF et le Fonds National de la Recherche Scientifique (FNRS) de Belgique.

\section{Références}

Alirol, E., Getaz, L., Stoll, B., et al. (20l I) Urbanisation and infectious diseases in a globalised world. The Lancet infectious diseases, I I(2), I3 I$14 \mid$.

Barreto, ML., Genser, B., Strina, A., et al. (2007) Effect of city-wide sanitation programme on reduction in rate of childhood diarrhoea in northeast Brazil: assessment by two cohort studies. The Lancet, 370(9599), 1622-1628.

Boschi-Pinto, .C, Lanata, CF., Mendoza, W., et al. (2006) Diarrheal Diseases. 2nd edition. In: Jamison, DT., Feacham, RGA., Makgoba, M., et al. (eds), Disease and Mortality in Sub-Saharan Africa, Washington (DC): World Bank, pp. I07-I 23.

Fewtrell, L. \& Colford, JM. (2004) Water, Sanitation and Hygiene: Interventions and Diarrhoea $A$ Systematic Review and Meta-analysis. Health, Nutrition and Population (HNP), Discussion paper, Washington D.C.: World Bank.

Filmer, D \& Pritchett, L. (200I) Estimating Wealth Effects Without Expenditure Data--Or Tears: An Application to Educational Enrollments in States of India. Demography, 38(I), II5-I32.

Gartner, A., Kameli, Y., Traissac, P., et al. (2007) Has the first implementation phase of the Community Nutrition Project in urban Senegal had an impact? Nutrition, 23(3), 219-228.
Heller, L. (1999) Who really benefits from environmental sanitation services in the cities? An intra-urban analysis in Betim, Brazil. Environment and Urbanization, II(I), I33-I44.

Hobcraft, J. (1993) Women's education, child welfare and child survival: a review of the evidence. Health Transition Review, 159-175.

Khatab, K. \& Kandala, N-B. (20II) Latent variable modelling of risk factors associated with childhood diseases: Case study for Nigeria. Asian Pacific Journal of Tropical Disease, I(3), I69-176.

Institute for Health Metrics and Evaluation (20/3) The Global Burden of Disease. Disponible sur http://vizhub.healthdata.org/gbd-compare/ [09.09.2015]

Linzer, DA. \& Lewis, JB. (20II) poLCA: An R package for polytomous variable latent class analysis. Journal of Statistical Software, 42(I0), I29.

McCutcheon, AL. (1987) Latent class analysis. Newbury Park, Calif.: Sage Publications.

Mosley, WH. \& Chen, LC. (1984) An Analytical Framework for the Study of Child Survival in Developing Countries. Population and Development Review, 10, 25-45.

Rasbash, J., Charlton, C., Browne, WJ., et al. (2009) MLwiN Version 2.I. Centre for Multilevel Modelling, University of Bristol.

Rego, RF., Moraes, LRS. \& Dourado, I. (2005) Diarrhoea and garbage disposal in Salvador, Brazil. Transactions of the Royal Society of Tropical Medicine and Hygiene, 99(I), 48-54.

Sambe-Ba, B., Espié, E., Faye, ME., et al. (20/3) Community-acquired diarrhea among children and adults in urban settings in Senegal: clinical, epidemiological and microbiological aspects. BMC infectious diseases, I3(580), I-7.

Scott, P., Cotton, A. \& Sohail Khan, M. (2013) Tenure security and household investment decisions for urban sanitation: The case of Dakar, Senegal. Habitat International, 40, 58-64.

United Nations Human Settlements Programme (2007) The state of the world's cities report 2006/7: the millennium development goals and urban sustainability: 30 years of shaping the habitat agenda. London: Earthscan.

Woldemicael, G. (20II) Diarrhoeal morbidity among young children in Eritrea: environmental and socioeconomic determinants. Journal of Health, Population and Nutrition, 19(2), 83-90. 